Economía, Sociedad y Territorio, vol. vı, núm. 21, 2006, 31-54

\title{
La privatización de la expansión metropolitana en Buenos Aires
}

Pedro Pírez*

\begin{abstract}
In this paper, we present a description of some concrete processes that made up the transformations in the metropolitan configuration and functioning in Buenos Aires in the 1990 s during the 20th Century. We are referring to components related to the impact of processes at the macro level (national and international) pertaining to restructuring and globalisation. We aim to understand the "forces" that mediate between the gl obal and the local arenas, identifying the actors and the relationships behind metropolitan changes as well as searching for the meaning that the city acquires and which is understood as "privatisation". In the first section of the paper, this concept is made explicit.
\end{abstract}

Keyw ords: metropolitan expansion, privatisation, urban soil, urban services, cl osed urbanisation.

\section{Resumen}

En este trabajo se presentan de manera descriptiva algunos procesos concretos que integran las transformaciones ocurridas en la configuración y funcionamiento metropolitanos de Buenos Aires en los años noventa del siglo xx. Se trata de componentes del impacto de procesos de nivel macro (internacionales y nacionales) propios de la reestructuración y la globalización. Se intenta conocer las 'fuerzas' que operan como mediaciones concretas entre lo global y lo local, identificándose los actores y las relaciones que están detrás de los cambios metropolitanos, y buscándose la significación que adquiere la ciudad, en este caso entendida como "privatización". En la primera parte del artículo se hace explícito ese concepto.

Palabras clave: expansión metropolitana, privatización, suelo urbano, servicios urbanos, urbanizaciones cerradas. 


\section{1. ¿De qué privatización hablamos? ${ }^{1}$}

Es ya un lugar común referir a la privatización de nuestras ciudades a partir de los cambios ocurridos (reestructuración y globalización) tanto en el ámbito internacional como en cada uno de los países de América Latina en las últimas décadas.

Para entender de qué se trata, es preciso recordar que esas ciudades, en general, se produjeron en un contexto de predominio de lógicas privadas. Esa producción se realizó fundamentalmente por procesos dirigidos y ejecutados por actores privados capitalistas que la orientaron, por un lado, por sus intereses particulares en el logro de beneficios en cada una de las operaciones (producción de suelo, de vivienda, de construcciones para actividades económicas, o para infraestructuras y equipamientos) y, por otro lado, por sus intereses generales, si puede utilizarse dicho término, para garantizar el funcionamiento de las actividades económicas en su conjunto y las condiciones de reproducción de la fuerza de trabajo.

El Estado intervino intentando limitar la subordinación de la producción urbana a los intereses particulares de cada productor. Ese intento se orientaría a mantener el Ilamado interés general (la ciudad como objeto de negocio versus la ciudad como ámbito de los negocios); a garantizar las condiciones de reproducción de la fuerza de trabajo, en su asentamiento y acceso a bienes y servicios; igualmente, a fortalecer su legitimidad en un sentido más amplio. La intervención estatal dependía en cada caso de los actores presentes, de sus relaciones y contradicciones. El resultado fue una tensión hacia resultados de integración social y territorial para el conjunto de las actividades económicas y de la población, en procesos de producción metropolitana privada.

Cuando se habla particularmente de privatización puede referirse tanto a los procesos de producción urbana (suelo y construcciones) como a sus resultados o productos. La privatización de la producción urbana implica su subordinación a decisiones de actores que se mueven en razón de una lógica de acumulación particular de capital y que se orientan por la obtención de ganancia en primer término, quedando en segundo lugar su posible vinculación con intereses generales, como sería la atención de intereses

${ }^{1}$ Este trabajo forma parte del proyecto UBAC y т SO 12 "La configuración urbana en la Región M etropolitana de Buenos Aires en los años noventa. Bases para la gestión". Una versión anterior fue presentada en el Seminario Buenos Aires M etropoli Locale. Racconti de una Citta en Transformazione, Universitá degli Studi, Roma Tre, mayo, 2005. 
o necesidades de otras unidades económicas o de la fuerza de trabajo o de la población en general.

La privatización de los productos se refiere a la capacidad de inclusión territorial y social de éstos y a la tendencia a dejar fuera de su consumo a segmentos importantes de la población. Esos efectos se relacionan, sin duda, con los procesos de producción, pero además tienen que ver con las condiciones de la población en general 0 de algunos de sus grupos en particular. Los cambios en esas condiciones pueden deberse tanto a modificaciones en el mercado de trabajo y en las relaciones de distribución social que dejan fuera del acceso a los bienes urbanos a sectores importantes de la población (desempleo, precarización del empleo, condiciones de pobreza, etc.), como a políticas estatales que no se hacen cargo de esos cambios o a la disminución de las políticas de apoyo a la reproducción de la población. ${ }^{2}$

Todo esto es parte de una particular relación mercado-Estado, donde el mayor predominio privado está asociado con tres situaciones: a) el Estado disminuye o debilita su intervención, con un sesgo en favor de la producción privada del espacio urbano; b) el Estado modifica el sentido de su intervención, orientándose no ya en razón de los "intereses generales" sino en favor de intereses económicos particulares, y c) la emergencia de un desequilibrio en la relación mercado-Estado por la transformación de los actores capitalistas, como puede ser un proceso de internacionalización, que altera su peso relativo frente al Estado y al resto de los actores sociales.

\section{La expansión metropolitana en Buenos Aires 2.1. Antecedentes}

Desde el comienzo de la expansión metropolitana de Buenos Aires, a principios del siglo xx, la producción urbana se diferenció en sus dos territorios significativos: centro y periferia (Pírez, 1994).

En la ciudad central, la ciudad de Buenos Aires, ${ }^{3}$ la producción se basó en algunas políticas del gobierno municipal, ${ }^{4}$ fundamen-

\footnotetext{
2 Existe una amplia literatura al respecto. Vale la pena mencionar los trabajos de Loic Wacquant (2001) sobre el impacto de esos procesos combinados en el caso de las sociedades avanzadas.

${ }^{3}$ La ciudad de Buenos Aires, Capital Federal desde 1880 y ciudad autónoma desde 1994, ocupa un territorio de $200 \mathrm{~km}^{2}$ desde 1887. En 2001 tenía casi 2.8 millones de habitantes.

${ }^{4}$ Entre 1882 y 1994, la ciudad de Buenos Aires fue gobernada por la M unicipalidad de Buenos Aires, que dependía del gobierno federal. El presidente elegía al ejecutivo
} 
talmente el diseño de un plano que estableció una cuadrícula completa para toda la ciudad (1889-1904) y orientó una ocupación relativamente homogénea del territorio desde principios de siglo (Gorelik, 1998:24); la producción de obras públicas que dieron una fuerte centralidad, ${ }^{5}$ y la producción privada con regulación municipal de los servicios, especialmente energía eléctrica y transportes (ferrocarriles, tranvías y autobuses), así como la producción y gestión estatal de la red de agua y saneamiento. Sobre esa base se expandió la ciudad incorporando suelo producido privadamente, estando a cargo de los ocupantes la iniciativa para completar el medio construido (Dupuy, 1987; Pírez, 1994 y 1999). El centro metropolitano se configura con una calidad urbana relativamente alta: cobertura de servicios y normas de uso del suelo que, más allá de sus limitaciones, sientan la base de una producción con capacidad de integración social.

Por otra parte, la periferia metropolitana, ${ }^{6}$ que en la primera mitad del siglo xx creció por la expansión de las recientes clases medias ${ }^{7}$ y luego, a partir de los años cuarenta, de los sectores populares ${ }^{8}$ (en gran medida obreros industriales), contó con una infraestructura que quedó rezagada respecto de la expansión territorial, y casi sin normas que regularan la producción del suelo. De todas formas, el mercado del suelo, por medio del llamado "loteo popular" (Clichevsky, 1990), permitió el asentamiento regular de la población que se incorporaba a la ciudad. D esde los años cuarenta, con la estatización de los servicios urbanos, si bien la cobertura siguió siendo limitada, las tarifas bajas, y sobre todo la 'permisibilidad' de los consumos clandestinos, redundaron en una relativa integración, aunque de baja calidad urbana.

El resultado fue una ciudad que integraba en forma regular, aunque segregada y muy desigual, a la población de menores recursos. Esos procesos se modificaron en las últimas décadas.

local (intendente), mientras que el Congreso delegaba en el Concejo Deliberante, de elección popular, atribuciones locales. Actualmente, como ciudad autónoma, elige al jefe de gobierno y a la Legislatura.

5 Paradigmáticamente, la Avenida de M ayo.

${ }^{6}$ Integrada por municipios de la provincia de Buenos Aires que, en número creciente en el tiempo, suman unos 30 en 2001, con una población de casi nueve millones de habitantes.

${ }^{7}$ Formadas por los inmigrantes de ultramar que se integran económica y socialmente (Lattes y Lattes, 1992; Germani, 1987). 1992).

${ }^{8} \mathrm{~A}$ partir de las migraciones internas y de algunos países limítrofes (L attes y Lattes, 


\subsection{La configuración metropolitana en los noventa}

En la Argentina, las políticas coherentes con el proceso de reestructuración económica9 ${ }^{9}$ comenzaron con el gobierno militar implantado con el golpe de marzo de 1976. Se revertió el modelo de industrialización por sustitución de importaciones, provocando desempleo y fuerte deterioro de la distribución económica. El gobierno central se retiró de las políticas sociales más importantes en infraestructura (agua y saneamiento y distribución eléctrica), salud y educación, descentralizando en primer lugar hacia los gobiernos provinciales y luego hacia los municipios. Se inició un proceso general de disminución del Estado, ${ }^{10}$ con la baja de su gasto social.

Esas políticas se consolidaron y ampliaron durante el gobierno del presidente $C$ arlos $S$. M enem en los años noventa, en un proceso de desregulación y apertura de la economía.

A continuación mencionamos los aspectos más relevantes para nuestro razonamiento.

En un contexto de reforma y achicamiento del aparato estatal, con disminución de funciones y recursos, se transfirieron tareas a otros niveles estatales (descentralización) y hacia empresas privadas (privatización). Se modificó el papel del Estado, con un peso mucho menor en la aplicación de políticas y recursos para la población de bajos ingresos y una creciente orientación hacia la promoción de los negocios en diferentes campos (entre ellos el inmobiliario). Esos años mostraron la presencia cada vez mayor del capital transnacional en la economía del país, y particularmente el peso del capital financiero, así como el predominio económico del sector terciario (finanzas y servicios) y el crecimiento de las actividades destinadas a la exportación (productos agropecuarios y energéticos) (Ciccolella, 2000). Los cambios provocados en el mercado de trabajo fortalecieron relativamente algunos pocos puestos de gran calificación y remuneraciones muy altas (generalmente en el sector terciario) y deterioraron las ocupaciones industriales, promovieron el desempleo y el aumen-

9 Tres son sus rasgos fundamentales: a) apropiación por el capital de cada vez mayor excedente, aumentando la productividad y la explotación, con reestructuración del proceso de trabajo y del mercado laboral; b) cambio en el modelo de intervención estatal, en detrimento de la legitimación y la redistribución, y c) internacionalización de los procesos económicos para incrementar rentabilidad y abrir mercados (Castells, 1995: 52-57).

${ }^{10}$ El lema repetido hasta el cansancio fue: "Achicar el Estado es agrandar la N ación". 
to de las desigualdades en la distribución del ingreso, con una creciente polarización. De allí la emergencia de una clase media alta con fuertes ingresos y gran capacidad de consumo y, como contraparte, el aumento de la población bajo la línea de pobreza e indigencia, con brechas cada vez más amplias entre ambos sectores, y el debilitamiento de la clase media tradicional. Entre 1991 y 2002 , la diferencia entre los recursos recibidos por $20 \%$ de la población con menores ingresos y $20 \%$ de la población con mayores recursos pasó de 9.2 a 14.5 veces. Entre 1992 y 2001, la población bajo la línea de pobreza e indigencia pasó de $27.4 \%$ a $52.4 \%$ (Pírez, 2004: 120 y 125).

En el ámbito metropolitano, todo ello supuso importantes modificaciones: se transformaron los actores que producían la ciudad. Por un lado, aparecieron nuevos actores económicos de gran poder y capacidad de decisión sobre la configuración metropolitana, con una verdadera 'superciudadanía' que superaba la capacidad de regulación y control de los usuarios y del Estado, en particular en los servicios urbanos (Pírez, 1994 y 1999; Pírez et al., 2003) y la producción del suelo. Coherentemente, desde el Estado se promovieron las actividades privadas en la producción urbana. ${ }^{11}$ Por otro lado, los grupos de menores ingresos vieron afectada su capacidad de reproducción por la disminución de la oferta estatal de bienes urbanos (suelo, vivienda, servicios), ${ }^{12}$ en momentos en que crecían el desempleo y la pobreza, y comenzaron a producir de manera directa esos bienes, en una situación de 'subciudadanía' (Kovarik, 2000). Se produjo, también, un cambio en los patrones de asentamiento residencial de las clases media alta y media, en un proceso de suburbanización a partir del consumo de 'urbanizaciones cerradas'.

El resultado, en términos agregados, fue una mayor privatización de la ciudad en el doble sentido mencionado. Para estudiar ese proceso observaremos lo que sucedió con la producción de la expansión metropolitana.

\footnotetext{
${ }^{11}$ El Estado hace posibles operaciones inmobiliarias nuevas: la gran cantidad de excepciones al Código de Planeamiento en la entonces $M$ unicipalidad de Buenos Aires y la promoción de grandes proyectos inmobiliarios desde el gobierno federal 0 en asociación con la M unicipalidad, el establecimiento de la Corporación Antiguo Puerto $M$ adero, las condiciones para la construcción del proyecto Abasto, los proyectos frustrados de Retiro y Ciudad Judicial, las condiciones para la realización del emprendimiento de Santa M aría del Buen Ayre, etcétera.

12 Se trata de la práctica eliminación del Fondo $\mathrm{N}$ acional de la Vivienda (Fonavi), de la privatización del Banco H ipotecario y de los servicios urbanos.
} 


\section{La privatización de la expansión metropolitana}

En este análisis revisaremos la producción del suelo y de las infraestructuras urbanas atendiendo a la periferia, sin mencionar los procesos que ocurren en la ciudad central.

\subsection{La privatización de la producción del suelo metropolitano}

Diferenciaremos dos situaciones: suelo urbano para los sectores de menores recursos y residencia de las elites.

\subsubsection{El suelo de los pobres}

Desde los años cuarenta, la población de menores recursos se asentaba en "Ioteos populares" y "villas de emergencia" o "villas miseria".

Estas últimas fueron ocupaciones ilegales de tierras con la construcción de viviendas muy precarias sin ninguna urbanización. Se formaron por primera vez en los años treinta con inmigrantes desocupados (De la Torre, 1983), y ya en 1956 se censaron allí 112,350 personas que representaban 1.9\% de la población metropolitana (Yujnovsky, 1984).

Los "loteos populares" permitieron a la población de bajos ingresos acceder en forma legal ${ }^{13}$ a la tierra urbana producida en la periferia por promotores privados, en general sin infraestructura, a veces inundable, que se pagaba en cuotas mensuales y que era ocupada lentamente por casas autoconstruidas (Clichevsky, 1990: 5; Prévot y Schneier, 1990: 131). Todo ello fue posible por la falta de regulaciones y el contexto de amplia incorporación al mercado de trabajo y de redistribución económica.

Las políticas del gobierno militar iniciado en 1976 alteraron esas condiciones, más allá de medidas generales que disminuyeron la orientación pública de la producción de la ciudad. ${ }^{14}$ En 1976 se suspendió la subdivisión de loteos y en 1977 se reguló el ordenamiento territorial y el uso, subdivisión, ocupación y equipamiento del suelo. Fueron obligatorios requisitos de infraes-

${ }^{13}$ Entre 1947 y 1960, los propietarios aumentaron de 43 a 67\% (Clichevsky, 1999)

${ }^{14}$ Eliminación del congelamiento de arrendamientos urbanos; indexación de los contratos de compra-venta de tierras, entre ellos los loteos populares; erradicación autoritaria y represiva de las villas miseria de la ciudad de Buenos Aires (Pírez, 1994). 
tructura básica para los loteos, dimensiones y equipamientos mínimos (D ecreto Ley 8912). El efecto más importante fue la limitación de los loteos y un fuerte encarecimiento de la tierra (Clichevsky, 1999).

Esas normas se sumaron al impacto de las políticas económicas que incrementaban la desocupación y disminuían el salario. La consecuencia fue la crisis y desaparición del submercado legal de loteos populares. La ciudad dejó de ofrecer suelo legal a los pobres urbanos y éstos debieron resolver por sí mismos esa necesidad. En los años ochenta comenzó la toma ilegal de tierras públicas y privadas y la formación de "asentamientos" 15 (Izaguirre y Aristazabal, 1988; M erklen, 1991 y 2000). La población excluida se organizó para ocupar (ilegalmente) tierras y construir fraccionamientos, con base en una previa planificación que definía áreas privadas y públicas a ser desarrolladas. Se sustituyó la acción estatal por una planificación urbana popular orientada a la satisfacción directa de la necesidad de quienes se organizan, planifican y construyen.

Todo eso incrementó el deterioro del hábitat popular, tanto en términos formales (localización ilegal en tierra y edificios privados o públicos, loteos clandestinos, etc.) como urbano-ambientales (áreas inundables y contaminadas, sin infraestructuras ni servicios, pésima accesibilidad, sin conexión con áreas centrales, etcétera).

En la década del ochenta se iniciaron algunas políticas orientadas fundamentalmente a la regularización dominial (Clichevsky, 1999). Atendieron en forma limitada el problema sin hacerse cargo de las condiciones económicas de la población, que veía aumentar sus gastos: pago de la tierra, impuestos prediales y tasas municipales, y costos de los servicios urbanos privatizados. Luego de la crisis de fines del año 2001, los municipios metropolitanos se incluyeron en el Programa de M ejoramiento de Barrios aplicado con recursos del Banco Interamericano de Desarrollo y con fondos nacionales. Actualmente se están ejecutando proyectos que incluyen a 3,200 familias en seis de esos municipios. ${ }^{16}$

La ausencia de procedimientos legales para resolver la necesidad de suelo consolidó un universo irregular en la periferia y promovió la expansión metro politana en búsqueda de tierras para invadir o con precios accesibles para las mínimas ofertas públi-

\footnotetext{
${ }^{15}$ Así llamados para diferenciarlos de las villas miseria y de su estigmatización social.
}

${ }^{16} \mathrm{Cfr}$. www.promeba.org.ar/avance/index800.html, 11 de noviembre de 2005. 
cas. Para fines de los años noventa, más de un millón de personas vivían en condiciones de irregularidad legal y de precariedad urbano-ambiental, ${ }^{17}$ mientras continuaban las invasiones (Clichevsky, 1999).

\subsubsection{La residencia de los ricos}

En los años noventa se inició un proceso de suburbanización de clases medias altas y medias que, en un contexto de fuerte desempleo, pobreza y exclusión social, se concretó en las llamadas "urbanizaciones cerradas". En este texto analizamos ese proceso solamente como parte de una planificación privada de la producción del suelo metropolitano asociada con:

- Cambios en las condiciones de la demanda. Como consecuencia de las modificaciones económicas y del mercado de trabajo creció y se fortaleció una clase media alta con gran capacidad de consumo. ${ }^{18}$ Esos grupos, insertos en las actividades más ligadas al mercado internacional (finanzas, servicios a las empresas, etc.), desarrollaron un estilo de vida donde el consumo ostentoso se convirtió en elemento de identidad. ${ }^{19}$ Dos bienes eran esenciales: el automóvil y la residencia. Al mismo tiempo, la producción pública de la ciudad, crecientemente debilitada, se alejó cada vez más de sus demandas.

- Cambios en las condiciones de la producción. Con el fin de los loteos populares quedaron reservas de suelo que en un primer momento se destinaron a country clubs y a cementerios jardín (Prévot y Schneier, 1990: 124), y luego a la producción de urbanizaciones cerradas. Todo eso en un contexto de debilidad normativa: ausencia de normas metropolitanas y limitación a la aplicación por los municipios de la Ley 8912 mencionada.

Cambian los actores que producen suelo, se profesionalizan y concentran, e intervienen capitales y tecnologías extranjeros (M ignaqui y Szajnberg, 2003). Se desarrolla una gran campaña

${ }^{17}$ Aproximadamente $10 \%$ de la población del área.

${ }^{18}$ Entre 1998 y 2001, 20\% de la población de mayores ingresos recibió, respectivamente, $70.5,70.7,69.7$ y $70.6 \%$ del total de los ingresos metropolitanos.

${ }_{19}$ Proceso trabajado para los países avanzados, por ejemplo, por Saskia Sassen (1991). 
de marketing ${ }^{20}$ que fortalece el prestigio de la residencia suburbana cerrada como parte de un estilo solamente accesible para quienes tienen altos ingresos.

La transformación de la red de accesos a la ciudad de Buenos Aires, privatizada a comienzos de los años noventa, permitió conectar rápidamente al centro metropolitano con la periferia más lejana, junto con el gran crecimiento del uso de automóviles. ${ }^{21}$

Como resultado se produjo un ámbito urbano segregado y altamente vigilado que introdujo discontinuidad en el tejido urbano, fragmentando el espacio metropolitano, con una frontera que no puede ser atravesada salvo por quienes están autorizados (los propietarios y sus invitados). Ese territorio cerrado ofrece infraestructuras, servicios urbanos (redes de electricidad, gas, teléfono, internet, pavimentos, alumbrados, mantenimiento, espacios verdes, vigilancia), áreas comerciales y recreativas, oficinas, servicios educativos y centros médicos y culturales. Producen un excluyente fragmento privado de ciudad de alta calidad. Para fines de siglo habría entre 300,000 y 500,000 personas residiendo en unas 400 unidades de esa naturaleza, más de 130 en el municipio de Pilar, en donde cerca de $30 \%$ de la superficie es de acceso restringido (J anoschka, 2003). Lo anterior es particularmente significativo en las Ilamadas "ciudades pueblos" o "megaurbanizaciones cerradas". Son urbanizaciones de entre 400 y 1,600 hectáreas, que incluyen a muchos barrios, con más de 2,000 viviendas y con una población potencial de cerca de 200 mil personas en la más grande (Janoschka, 2002; Vidal-Koppmann, 2004). ${ }^{22}$

Al observar la producción de esos territorios se percibe la configuración de un proceso privado de planificación que introduce una dura racionalidad en el emprendimiento y olvida al resto de la ciudad en la cual se asienta.

Debe recordarse la ausencia de una planificación ${ }^{23}$ territorial metropolitana estatal que reconozca dicha unidad territorial de

${ }^{20}$ Un buen ejemplo es la publicación en los dos diarios más importantes del área metropolitana (Clarín y La N ación) de suplementos semanales destinados a ese mercado, cubriendo además de lo inmobiliario, información sobre construcciones, insumos para la vida suburbana, con información sobre la vida social, deportiva y cultural de esos "barrios".

21 Unos tres millones de automóviles representan 37\% de los viajes en 1997. En 1970, los viajes en ese medio ascendían a $15.4 \%$.

${ }^{22}$ Actualmente se desarrollan ocho en el Área M etropolitana de Buenos Aires (Vidal-Koppmann, 2004).

${ }^{23}$ Entendemos planificación como el intento de introducir una orientación pública (integradora) por medio del establecimiento de parámetros para el comportamiento de 
funcionamiento. Podría suponerse que la ley provincial 8912 tendría ese carácter, pero es una norma 'abstracta' hecha para la totalidad de los municipios de esa provincia que ellos deben aplicar. Por lo demás, se reforma en esos años para permitir este nuevo uso de suelo (M ignaqui y Szajnberg, 2003). En consecuencia, los municipios tienden a dirimir el asunto en relación con sus intereses particulares, económicos y políticos. Los municipios metropolitanos periféricos (como Pilar y Tigre), con menor ocupación del suelo, definen como ventajosas las propuestas de los productores privados y facilitan sus proyectos. El resultado es el peso creciente de los actores privados en las decisiones de producción inmobiliaria (Janoschka, 2002; N úñez et al., 1998). Como dice uno de ellos, "realmente hoy lo que permite el capital privado es determinar y regular normas" (citado por Jacky y Triegerman, 2000).

Esas urbanizaciones son el resultado de una planificación privada que sustituye a la inexistente o muy débil planificación estatal. La ciudad se produce en una 'racionalización' mercantil de operaciones individuales. Ello implica una fuerte planificación 'interna' de los componentes de cada operación y un sistema de control de su cumplimiento, con la finalidad de aumentar la calidad del producto inmobiliario y su rentabilidad.

Esa operación se limita a los territorios privados donde "se trata de planificar cuidadosamente una ciudad desde su punto cero" (Clarín, 30/10/99). Como apunta el titular de la empresa a cargo de Nordelta: ${ }^{24}$

[... ] la ciudad se diseña teniendo en cuenta el equilibrio entre espacios verdes, agua y zonas urbanas; el paisaje, la forma de las calles, la localización de los barrios, colegios, universidades, clubes, zonas comerciales... Se le da al entorno una armonía estética y urbanística, con distintas densidades de población y una adecuada distribución del tráfico.

D e esa forma, continúa el periodista que lo entrevista, se evitarán los problemas de las ciudades, donde a causa de un desarrollo desordenado, el crecimiento de la población se dispara a niveles impensados y produce inconvenientes como embotellamiento de tránsito (Clarín, 30/10/99).

los actores estatales y no estatales, sea que se haga desde intentos tradicionales, como la imposición técnico-política de un modelo, o bien con formas no tradicionales, que integren en la formulación y ejecución a los actores de la sociedad civil (Klink, 2003).

${ }^{24}$ La mayor de esas megaurbanizaciones. 
Lo anterior muestra una operación intelectual que produce dos rupturas: a) la producción de una parte de la ciudad es transformada en 'la ciudad'. La parte se postula como el todo, escondiendo que sólo es posible 'dentro' de una ciudad real que le 'provee' de las condiciones (fuentes de trabajo, infraestructuras troncales, servicios generales) para su existencia, aunque sea 'autoexcluida', y b) el desorden urbano que resulta de la producción pública de la ciudad se reduce a las dificultades para la vida de los grupos de mayores ingresos. D esaparece la falta de condiciones para que la población de menores recursos pueda asentarse regularmente.

Como consecuencia, el 'resto' de la ciudad, la ciudad real que sustenta a las urbanizaciones cerradas, queda reducida a una suerte de limbo.

Estamos ante una planificación que niega la planificación urbana pública, desconociendo la posibilidad de introducir una racionalidad global, diferente de la del mercado. La ciudad se piensa cada vez más como resultado de la suma de operaciones privadas y sus 'intersticios'. O peraciones privadas que se implantan en un medio "caótico, Ileno de contradicciones y desventajas". M edio que no se percibe como objetivo de acción para su mejoramiento.

Si avanzamos un poco, podemos ver los componentes de esa planificación:

- Un sistema normas que emanan de un documento privado (contrato de compra-venta) y que se imponen como cláusulas de adhesión. Estrictas normas urbanas: de zonificación, uso del suelo y edificación. Lugares para residencia y para actividades. Los primeros diferenciados por estratos económicos con distinta cantidad y calidad de tierra, y por ende de precios; ${ }^{25}$ los segundos diferenciados por tipo de actividad. Normas de comportamiento social a las que se comprometen quienes acceden a esas urbanizaciones. Reglamentos de ética y de convivencia que funcionan como una suerte de derecho de admisión (o de exclusión). Esto muestra el uso de instrumentos

${ }^{25}$ En Pilar del Este, una 'ciudad cerrada' que prevé 12 barrios privados, "pueden convivir casas de 100 mil dólares con otras de un millón. Por eso nosotros preferimos tener sectores diferenciados. Pero por una cuestión urbanística, no social" (Jorge Álvarez, apoderado general de Inversiones Los Andes, S.A., en Clarín, 27/11/1999). ¿Cuál será esa cuestión urbanística no social? No será simplemente el fortalecimiento de la "natural" segregación social de nuestras ciudades. 
mercantiles para el logro de finalidades sociales que tienden a consolidar la identidad de cada proyecto.

- Amplia oferta de infraestructuras y servicios de alta calidad vinculados con la "reproducción de la población" que hace innecesario salir del territorio cerrado, salvo para ir a trabajar.

- Subordinación de recursos naturales a la valorización del emprendimiento, tanto por la promoción de la expansión metropolitana con un uso dilapidatorio del suelo, como por una utilización arbitraria del suelo ocupado, como sucede con la 'polderización' de áreas sin control público ni estudios del impacto sobre los residentes anteriores.

- 'Gravámenes' financieros para los residentes destinados a sostener la producción y mantenimiento de infraestructura y servicios. Esa suerte de impuestos privados tienen también la función de diferenciar económicamente los territorios, en primer lugar en relación con el afuera, y luego entre los diferentes "adentros".

- La existencia de sanciones por incumplimientos de normas que pueden llegar a multas y que son decididas por la conducción privada de la urbanización.

- Un sistema de toma de decisiones que somete a la población y que se estructura (administrativamente) con limitada participación de los residentes y el predominio formal de los propietarios originales del emprendimiento (gobierno privado-mercantil).

En suma, se reproduce en la producción de la ciudad la lógica global del mercado: competencia desordenada frente a una fuerte racionalidad (planificación) en cada unidad individual, orientada al cumplimiento de condiciones de comercialización para el logro de calidad para algunos.

\subsection{La privatización de las infraestructuras urbanas}

Desde la década de 1940, los servicios urbanos del Área M etropolitana de Buenos Aires quedaron a cargo de empresas públicas del gobierno federal (Pírez, 1999). Para fines de los años ochenta, el deterioro de esas empresas era tal (en gran medida debido a la mala gestión de la dictadura militar) que mostraban baja cobertura, problemas financieros e incapacidad de realizar inversiones, mala calidad y, en algunos casos, corrupción en sus relaciones con sus sindicatos y empresas privadas que proveían 
bienes y servicios. Recordemos que esa gestión fue permisiva con los consumos clandestinos de la población de bajos recursos, evitando en cierta medida su exclusión del servicio.

La asociación de la crisis de las empresas con el déficit de las cuentas estatales y con la inflación fue la gran justificación para su privatización. Ésta se realizó velozmente a principios de los años noventa, transfiriéndose ${ }^{26}$ a empresas privadas la gestión de los servicios de infraestructura (teléfonos, electricidad y gas natural, agua y saneamiento), los transportes ferroviarios de superficie y subterráneos (los autobuses ya lo eran), y la infraestructura vial de acceso a la ciudad. ${ }^{27}$ La privatización modificó a los actores y sus relaciones: el Estado se excluyó como objeto de relaciones reivindicativas, reducido a asegurar el cumplimiento de las relaciones de mercado, aun en su desigualdad. El ciudadano pasó a ser un cliente frente a las empresas. Los derechos de los usuarios sobre el servicio se diluyeron: el cliente solamente tiene derechos sobre el producto como un equivalente del precio que paga.

Como consecuencia de eso, los servicios dejaron de ser públicos en el sentido de un derecho, para ser considerados actividades económicas reguladas para lograr condiciones análogas a la competencia. Se convirtieron en una relación comercial privada. $M$ ejoraron en su calidad, por el aumento de su eficiencia, pero el incremento de las tarifas tendió a dejar fuera de ellos a la población de menores recursos. ${ }^{28}$ Las empresas de servicios, por lo general de origen internacional, se convirtieron en actores con gran peso económico y político. La debilidad de los marcos de regulación y de los organismos de control contribuyó a ello. En algunos casos se sumaron las presiones de los gobiernos de los países de origen.

Los principales efectos de ese proceso son:

- Acentuación de la concentración y exclusión económica y social. La regulación generó condiciones de muy limitado riesgo empresario y permitió exorbitantes tasas de rentabilidad (Aspiazu y Schorr, 2003: 21). Indexaciones basadas en la inflación de Estados Unidos, ${ }^{29}$ prohibidas por la Ley de Conver-

${ }^{26}$ En diferentes modalidades que no es relevante desarrollar ahora.

${ }^{27}$ Esta sección se basa en gran medida en Pírez, 1999; y Pírez et al., 2003, cap. 2.

${ }^{28}$ En las distribuciones de electricidad y gas natural se prohibieron los subsidios cruzados, con el argumento de que cada quien debe pagar su consumo.

${ }^{29}$ Entre septiembre de 1996 y diciembre de 2001, el Índice de Precios al Consumidor en Estados Unidos se incrementó en 12\%, mientras que en Argentina sufrió una deflación de 3\% (Aspiazu y Schorr, 2003: 24). 
tibilidad, dolarización y sucesivas renegociaciones, permitieron el crecimiento de las tarifas muy por encima de la inflación. ${ }^{30}$ En al gunos servicios como en el gas y la electricidad, las tarifas de los usuarios residenciales aumentaron más que las de los grandes usuarios; como consecuencia, y a diferencia de lo que sucedía en el modelo estatal, se produjo un sesgo regresivo, con lo que parecía un subsidio implícito en favor de los grandes consumidores industriales (Aspiazu y Schorr, 2003: 19). Dado el incremento de la tarifas y el fin de los subsidios y de los mecanismos permisivos que existían en la gestión estatal, la población de bajos recursos vio dificultado su acceso y permanencia en los servicios (Pírez, 2000). Eso no solamente afectó a los usuarios individuales, sino que alteró las condiciones urbanas para el desarrollo de las actividades, "afectando negativamente la competitividad de numerosos sectores económicos -en particular, aquellos, con predominancia productiva de pequeñas y medianas empresas, vinculados a la elaboración de bienes transables- y la distribución del ingreso" (Aspiazu y Schorr, 2003: 38).

Como resultado, las empresas de servicios recibieron ganancias sumamente altas. ${ }^{31}$

- La definición de componentes importantes de la planificación urbana por parte de las empresas. Se les transfirió a las empresas la capacidad de definir la política y sobre todo la planificación de los servicios, teniendo un papel clave en la configuración urbana (Pírez et al., 1999). Cada empresa toma decisiones de acuerdo con sus necesidades mercantiles, sobre qué territorios cubrir, qué procesos desarrollar y en qué orden hacerlo. Se atienden poblaciones y territorios, y se realizan las operaciones que resultan de mayor y más rápida rentabilidad (por ejemplo, la expansión de la red de agua y no la de red de cloacas y el tratamiento del agua servida). El Estado no cumple un rol de planificador. La posibilidad de ampliar el servicio surge del mercado, de las relaciones entre las empresas prestadoras y los usuarios, lo cual supone un poder de decisión privado. Como consecuencia, dependen de las deci-

${ }^{30}$ La tarifa de agua, por ejemplo, se incrementó $88.2 \%$ entre mayo de 1993 y enero de 2002, mientras que el Índice de Precios al Consumidor aumentó 7.3\% (Aspiazu y Schorr, 2003: 32).

${ }^{31}$ Entre 1993 y 1997, la cúpula empresaria argentina obtuvo una rentabilidad promedio de $5 \%$, mientras que la distribución del gas logró una de $11.6 \%$, y las telefónicas, una de 15.6\% (Pírez, 2000: 51). 
siones de esas empresas tanto el mercado del suelo como la calidad de vida urbana.

- La privatización de la relación con los usuarios. Esto es evidente en los procesos de negociación entre las empresas y los usuarios clandestinos que se desarrollaron sin intervención de autoridad gubernamental alguna y con manejo discrecional, pudiendo convenir soluciones diferentes para casos análogos. La participación gubernamental ocurre solamente cuando emerge un conflicto político (Pírez, 2000). Tal parece ser el nuevo rol del Estado como regulador: ejercer el poder de policía sobre las adjudicatarias de los servicios, conformando un escenario donde el ciudadano devenido en cliente está supeditado al poder de las empresas en una relación asimétrica.

Tal asimetría es muy clara al observar las políticas de las distribuidoras de electricidad con la población de bajos recursos que no puede cumplir con los pagos. Las empresas prefieren no cortar el servicio por los costos que ello supone y porque es muy probable la posterior conexión clandestina. En consecuencia, negocian con esos usuarios, individual o colectivamente, diferentes formas de pago, sin la intervención de ningún organismo estatal. Así, cobran una parte de total facturado en cada periodo, adeudando los usuarios el resto que mes a mes se incrementa, sin que se sepa cuándo, cómo ni quién se hará cargo de esa deuda. ${ }^{32}$

\section{Conclusiones}

La producción de la periferia metropolitana de Buenos Aires vio modificar sus procesos y sus resultados como parte del efecto de las políticas vinculadas con la reestructuración económica, en un sesgo de incremento de la privatización en el doble sentido mencionado.

Por una parte, y como resultado general, se ampliaron las desigualdades socioeconómicas, con una fuerte concentración de los ingresos y el crecimiento de la pobreza.

De manera más específica, aumentó la participación del capital privado. Esa ampliación se debió a la participación de grandes actores económicos con conexiones internacionales (capital, dirección, tecnología, financiamiento) que organizaron operacio-

32 En algunos casos utilizan mecanismos de "energía prepaga" por medio de tarjetas para las familias, y cabinas de recarga. 
nes altamente rentables. La producción urbana (suelo, construcciones, servicios) tendió a organizarse por la ganancia de cada operación particular, contribuyendo al proceso de concentración económica indicado.

El asentamiento en la ciudad quedó librado al esfuerzo de cada familia o grupo social. Ya no dispuso de lugares para los pobres. La periferia metropolitana se segmentó y segregó más. Se ampliaron las diferencias entre los respectivos medios construidos y se profundizaron las distancias sociales.

El espacio de la expansión metropolitana quedó marcado por la presencia dominante de dos lógicas 'no estatales': la de los sectores excluidos del mercado formal, en la satisfacción directa de su necesidad; y la producción privada capitalista para los grupos de mayores ingresos. Como consecuencia, se dio una clara diferenciación entre cada territorio particular producido y el resto de la periferia. Los territorios producidos por la lógica de la necesidad, pese al intento de adecuación formal a la urbanización regular (lotes, calles, zonas para usos públicos, etc.), logran solamente muy baja calidad, a la vez que se insertan en medios hostiles, donde difícilmente se producen las infraestructuras y los servicios necesarios para su funcionamiento.

Los ámbitos internos de la producción capitalista, las "urbanizaciones cerradas", parecen reproducir lo que Robert Fishman (1987) Ilamó bourgeois utopias: alta calidad del hábitat, segregación basada en la identidad social para proteger a la familia separándola de la amenaza de la vida urbana y de los otros, particularmente los pobres, viviendo en contacto con la naturaleza. La ciudad restante solamente parece percibirse como condición de los flujos que permiten funcionar a esas urbanizaciones. Su ocupación se concreta en la conectividad y su consecuencia de acceso a lugares de trabajo y de consumo calificado, y está garantizada por la presencia de las empresas privatizadas de servicios (accesos viales y ferrocarriles, en particular). Por lo demás, ese 'resto' parece no existir. Esa calificación implícita de "tierra de nadie" es, paradójicamente, coherente con la ocupación (ilegal) de suelo por familias de muy bajos ingresos para construir sus "asentamientos" de ínfima calidad urbana.

De al guna manera, en ambas situaciones el 'resto' de la ciudad parece quedar librado a las fuerzas de cada uno de los actores sociales o económicos que lo ocupan (reproduciendo las relaciones de mercado). Las empresas que producen los servicios de infraes- 
tructura operan y consolidan allí su capacidad de decidir la configuración metropolitana, fortaleciendo su orientación privada en la búsqueda de ganancias particulares. Los sectores populares, intentando vencer su hostilidad, aplican la misma lógica de necesidad para superar su aislamiento, produciendo servicios en ese 'resto'. ${ }^{33}$

Se produce un efecto que puede considerarse paradójico. La expansión metropolitana resultante tiende a localizar con cierta cercanía física a los asentamientos precarios y a las urbanizaciones cerradas de las elites. La periferia metropolitana parece conformar un conjunto descentralizado de unidades territoriales homogéneas hacia dentro y heterogéneas entre sí. Eso supone un doble movimiento: pérdida de la heterogeneidad de la ciudad clásica que hacía posible los contactos entre grupos diferentes (por la segregación y el 'cierre'), y la emergencia de una nueva heterogeneidad (con grandes distancias socio-económicas) en una suerte de articulación de fragmentos, en lo que hemos llamado "microfragmentación" (Pírez, 2004: 123). Los dos extremos de la pirámide social que ocupan la periferia quedan colocados muy cerca en el espacio, siendo posibles sus relaciones: servicios sin calificación, aprovechamiento de residuos sólidos y otros como, por qué no, delitos. N o es ya la heterogeneidad de la integración. Es, por el contrario, la heterogeneidad de la exclusión.

D ebe tenerse en cuenta que las condiciones de consumo de la población, que dependen del mercado de trabajo y de las relaciones de distribución económica, son fundamentales para definir la significación incluyente o excluyente de los productos urbanos. La exclusión supone una sociedad que no garantiza a todos sus miembros la adecuación entre ingresos y costos de su reproducción. De allí que, por ejemplo, el incremento de las tarifas de los servicios no tiene los mismos efectos en una ciudad donde todos obtienen en el mercado de trabajo los recursos suficientes, que en otra (como en Buenos Aires) donde es cada vez mayor la cantidad de población que no logra obtener esos recursos. I gualmente, la obligación de mejorar la calidad del suelo que se produjo impuesta en 1977, cuando sus demandantes no podían afrontar los mayores costos, tuvo el resultado, paradójico, de eliminar el mercado formal del suelo para la población de bajos ingresos.

${ }^{33}$ Es el caso de la cooperativa de transporte El Colmenar, que permite a los habitantes pobres de la periferia del municipio de M oreno ir a lugares de trabajo y de consumo de servicios. 
En suma, los cambios ocurridos en la sociedad argentina y en el Área M etropolitana de Buenos Aires, desde mediados de los años setenta y particularmente en los noventa del siglo pasado, caracterizados por la privatización de la producción y de los productos, dieron lugar a una ciudad más desigual, concentradora y segregada.

\section{Bibliografía}

Aspiazu, Daniel y M artín Schorr (2003), Crónica de una sumisión anunciada. Las renegociaciones con las empresas privatizadas bajo la Administración Duhalde, Instituto de Estudios sobre Estado y Participación-Siglo xxı EditoresFacultad Latinoamericana de Ciencias Sociales, Buenos Aires.

Castells, M anuel (1995), La ciudad informacional. Tecnologías de la información, reestructuración económica y el proceso urbano-regional, Alianza Editorial, M adrid.

Ciccolella, Pablo (2000), "G randes inversiones y dinámicas metropolitanas. Buenos Aires: ¿Ciudad global o ciudad dual del siglo xxı?", Mundo Urbano, septiembre, 5.

Clichevsky, N ora (1990), "Política urbana y sector inmobiliario", en N ora Clichesky, M arie France Prévot Schapira y Graciela Schneier, Loteos populares, sector inmobiliario y gestión local en Buenos Aires. El caso del Municipio de Moreno, Cuadernos del Ceur 29, Centro de Estudios Urbanos y Regionales (CEUR), Buenos Aires.

(1999), Políticas de regularización en Argentina: entre la euforia y la frustración", mimeo, Buenos Aires.

(2003), "Territorios en pugna: Ias villas de Buenos Aires", Ciudad y Territorio: Estudios Territoriales, M adrid, verano-otoño, xxxv (136-137): 347-374.

De la Torre, Lidia (1983), "La ciudad residual", en José Luis Romero y Luis Alberto Romero, Buenos Aires, historia de cuatro siglos, t. II, Editorial Abril, Buenos Aires. 
Dupuy, Gabriel (1987), La Crise de réseaux: d' infraestruture: le cas de Buenos Aires, Ecole $\mathrm{N}$ ationale des Ponts et Caussées, Université Paris XII, París.

Fishman, Robert (1987), Bourgeois Utopias. The Rise and Fall of Suburbia, Basic Books, N ueva York.

Germani, Gino (1987), Estructura social de la Argentina. Análisis estadístico, Ediciones Solar, Buenos Aires.

Gorelik, Adrián (1998), La grilla y el parque. Espacio público y cultura urbana en Buenos Aires, 1887-1936, Universidad $\mathrm{N}$ acional de $\mathrm{Q}$ uilmes, Bernal.

Izaguirre, Inés y Zulema Aristazabal, (1988), Las tomas de tierras en la Zona del Sur del Gran Buenos Aires: un ejercicio de formación de poder en el campo popular, mimeo, Buenos Aires.

Jacky, Ricardo y Daniela Tregierman (2000), El impacto de las urbanizaciones cerradas sobre la gestión departamental, monografía presentada en el Seminario Gobiernos M unicipales y Ciudades $\mathrm{M}$ etropolitanas, Carrera de Ciencia Política, Facultad de Ciencias Sociales de la Universidad de Buenos Aires, Buenos Aires.

Janoschka, M ichael (2002), “El nuevo modelo de la ciudad latinoamericana: fragmentación y privatización", EURE, Santiago, 28(85): 11-20.

(2003), "N ordelta - ciudad cerrada. El análisis de un nuevo estilo de vida en el Gran Buenos Aires", Scripta N ova, Revista Electrónica de Geografía y Ciencias Sociales, Barcelona, vII (146). http://www.ub.es/geocrit/sn/sn146(121).htm.

Kilnk, Jeroen (2003), Perspectivas recientes sobre la organización metropolitana. Funciones y gobernabilidad, en el taller La Gobernabilidad de las Aglomeraciones de América Latina y el $C$ aribe, Banco Interamericano de Desarrollo, Washington, D.C, 4-5 de diciembre. 
Kowarick Lucio (2000), Escritos urbanos, Editora 34, Sao Paulo.

Lattes, Alfredo y Zulma Rechini de Lattes (1992), "Auge y declinación de las migraciones en Buenos Aires", en Jorge J orrat y Ruth Sautu (comps.), Después de Germani. Exploraciones sobre estructura social Argentina, Paidós, Buenos Aires, pp. 176-196.

M erklen, Denis (1991), Asentamientos en La Matanza. La terquedad de lo nuestro, Catálogos, Buenos Aires.

(2000), “Vivir en los márgenes: la lógica del cazador. N otas sobre sociabilidad y cultura en los asentamientos del Gran Buenos Aires hacia fines de los noventa", en M aristela Svampa (ed.), D esde abajo. La transformación de las identidades sociales, Biblios-Universidad Nacional General Sarmiento, Buenos Aires, pp. 81-119.

M ignaqui, Iliana y Daniela Szajnberg (2003), "Tendencias en la organización del espacio residencial en la Región M etropolitana de Buenos Aires en los noventa", en Rodolfo Bertoncello et al. (comps.), Procesos territoriales en Argentina y Brasil, Instituto de Geografía de la Universidad de Buenos Aires, Buenos Aires.

N uñez, Teresa, Silvia Jankilevich, Fernando Brunstein y Alejandro Pelfini (1998), "Agentes públicos y privados en la construcción de un desarrollo sustentable. Tigre: las dos caras de la ciudad global", Documento de Trabajo, Universidad de Belgrano, Buenos Aires.

Pírez, Pedro (1994), Buenos Aires metropolitana. Política y gestión de la ciudad, Centro Editor de América Latina, Buenos Aires.

(1999), "Gestión de servicios y calidad urbana en la ciudad de Buenos Aires", EURE, Revista Latinoamericana de Estudios Urbano Regionales, Santiago de Chile, xxv (76): 125-139. 
(2000), Servicios urbanos y equidad en América L atina, un panorama con base en algunos casos, Cepal, serie M edio Ambiente y Desarrollo 26, Santiago de Chile.

(2004), "La configuración metropolitana de Buenos Aires: expansión, privatización y fragmentación", Realidad Económica, Buenos Aires, 208.

, N atalia Gitelman y Juliette Bonnafè (1999), "Consecuencias políticas de la privatización de los servicios urbanos en la Ciudad de Buenos Aires", Revista Mexicana de Sociología, M éxico, año LXı (4).

, Elías Rosenfeld, Jorge L. Karol y Gustavo A. San Juan (2003), El sistema urbano-regional de redes de servicios e infraestructuras. Materiales para su estudio, Editorial dela Universidad de La Plata, La Plata.

Prévot Schapira, M arie France y Graciela Schneier (1990), “Poder local y gestión de la tierra", en N ora Clichesky, M arie France Prévot Schapira y G raciela Schneier, L oteos populares, sector inmobiliario y gestión local en Buenos Aires. El caso del Municipio de Moreno, Cuadernos del CEUR 29, CEUR, Buenos Aires.

Sassen, Saskia (1991), The Global City. N ew York, London, Paris, Princeton University Press, Princeton.

Vidal-Koppmann, Sonia (2004), Transformaciones socio-territoriales de la Región M etropolitana de Buenos Aires en la última década del siglo xx: la incidencia de las urbanizaciones privadas en la fragmentación de la periferia, ponencia presentada en el VIII Seminario Internacional de la Red Iberoamericana de Investigadores sobre Globalización y Territorio, Río de Janeiro, mayo.

Wacquant, Loic (2001), Parias urbanos. Marginalidad en la ciudad a comienzos del milenio, $\mathrm{M}$ anantial, Buenos Aires.

Yujnovsky, Óscar (1984), Claves políticas del problema habitacional argentino, 1955/1981, Grupo Editor Latinoamericano, Buenos Aires. 
Recibido: 21 de junio de 2005. Aceptado: 14 de noviembre de 2005.

Pedro Pírez es doctor en derecho y ciencias sociales por la Universidad $\mathrm{N}$ acional de Córdoba, donde se graduó como abogado y realizó un posgrado en sociología. Actualmente es investigador de carrera del Consejo $\mathrm{N}$ acional de Investigaciones Científicas y T écnicas (Conicet) con sede en el Centro de Estudios de Desarrollo y Territorio de la Escuela de Política y Gobierno de la Universidad Nacional de San M artín (Unsam). Es profesor titular de Gobiernos Locales en la Facultad de Ciencias Sociales de la Universidad de Buenos Aires, de Sociología Urbana en la $M$ aestría en Economía Urbana en la Universidad Torcuato Di Tella, y de Gestión M etropolitana en la M aestría en Desarrollo Local de la Unsam. Ha sido profesor en la Universidad $\mathrm{N}$ acional de Córdoba (Argentina); profesor-investigador en EI Colegio de M éxico, y profesor-investigador en el Doctorado en Urbanismo de la Universidad $\mathrm{N}$ acional Autónoma de $\mathrm{M}$ éxico. $\mathrm{H}$ a dictado cursos de posgrado en universidades de América Latina y de España. Lleva publicados unos 80 artículos en revistas académicas y compilaciones. Entre los últimos se encuentran: "Instituciones políticas y gestión urbana en el Área M etropolitana de Buenos Aires, Cuadernos Prolam/ usp, Sao Paulo, año 3, vol. 2, 2004; "Acumulación política municipal, fragmentación metropolitana y debilidad de lo público en Buenos Aires", en Juan de D. Pineda y Rita Grandinetti (coords.), La gestión pública en gobiernos local es. Experiencias latinoamericanas, Universidad N acional de Rosario-Universidad Autónoma de Tabasco, M éxico, 2004; “D escentralización, gobierno colegiado y gobernabilidad urbana. Algunas reflexiones", en M. Escolar, G. Badía y S. Frederic (eds.), Federalismo y descentralización en grandes ciudades. Buenos Aires en perspectiva comparada, Banco Ciudad-Prometeo Libros, Buenos Aires, 2004; "Buenos Aires metropolitana: expansión y privatización de una ciudad fragmentada", Anales Americanistas. Revista de Ciencias Sociales, 0 viedo, año 1, núm. 1, 2005. H a publicado ocho libros, entre los que pueden citarse: con G. Gamallo, Basura privada, servicio público, Centro Editor de América Latina, Buenos Aires, 1994; Buenos Aires Metropolitana. Política y gestión de la ciudad, Centro Editor de América Latina-centro, Buenos Aires, 1994; 
con E. Rosenfeld, J. L. Karol y G. A. San J uan, El sistema urbano regional de redes de servicios e infraestructuras. Materiales para su estudio, Universidad de La Plata, La Plata, 2003. 